\title{
Grammar or Serial Order?: Discrete Combinatorial Brain Mechanisms Reflected by the Syntactic Mismatch Negativity
}

\author{
Friedemann Pulvermüller ${ }^{1}$ and Ramin Assadollahi ${ }^{2}$
}

\begin{abstract}
If word strings violate grammatical rules, they elicit neurophysiological brain responses commonly attributed to a specifically human language processor or grammar module. However, an ungrammatical string of words is always also a very rare sequence of events and it is, therefore, not always evident whether specifically linguistic processes are at work when neurophysiological grammar indexes are being reported. We here investigate the magnetic mismatch negativity (MNN) to ungrammatical word strings, to very rare grammatical strings, and to common grammatical phrases. In this design, serial order mechanism mapping the sequential probability of words should neurophysiologically dissociate frequent grammatical phrases from both ungrammatical and rare grammatical strings. However, if syntax as a discrete combinatorial system is reflected, the prediction is that the rare, correctly combined items
\end{abstract}

\section{INTRODUCTION}

An intense debate focuses on the cortical grounding of language and grammar. Some cognitive scientists put forth that general principles of neural functioning, such as those of associative learning, apply to all cognitive domains alike and therefore provide an account of language processes, syntax and grammar included (Elman et al., 1996; Rogers \& McClelland, 2004). However, others have claimed that, in the human brain, grammar and syntax are dealt with by a discrete combinatorial system that processes rule-conforming strings of elements but rejects ungrammatical strings that violate syntactic rules, regardless of whether these strings have ever been perceived before (Ullman, 2001; Steedman, 2000; Pinker, 1994; Chomsky, 1957). These probabilistic and discrete combinatorial approaches make conflicting predictions on brain processes elicited by grammatical and ungrammatical strings. Clearly, common grammatical strings and uncommon ungrammatical strings should

\footnotetext{
${ }^{1}$ MRC Cognition \& Brain Sciences Unit, Cambridge, UK, ${ }^{2}$ University of Konstanz, Germany
}

group with the highly frequent grammatical strings and stand out against ungrammatical strings. Using magnetoencephalography as a measure of human brain activity, we replicated the previously reported syntactic mismatch negativity (SMMN), which distinguishes highly unfamiliar ungrammatical word sequences from common grammatical strings. Crucially, a significant interaction demonstrated that the sMMN specifically distinguished syntactic violations from common grammatical strings, but not uncommon from common grammatical word strings. This significant interaction argues in favor of a genuinely grammatical origin of the sMMN and provides direct neurophysiological evidence for a discrete combinatorial system for word and morpheme sequences in the human brain. The data are more difficult to explain in the context of serial order models that map co-occurrence probabilities of words.

be treated differently by the brain according to both approaches, as only the elements of the former would be assumed to be linked to each other-either by rules or by associative connections. However, when it comes to grammatical word sequences that only rarely occur, the predictions diverge: As associative learning principles imply that probabilities of co-occurrences of words are being mapped, they predict that rare grammatical strings group with rare ungrammatical ones. In contrast, the discrete processing system approach strongly predicts that, regardless of whether a string is rare or common, it should be its grammaticality or syntactic well-formedness that counts in terms of cognitive and brain mechanisms.

A critical test for the diverging predictions is offered by cortical electrophysiology. It is well known that ungrammatical word strings elicit neurophysiological signatures that do not show up, or appear in much reduced form, when well-formed strings are under processing. Among the syntactic brain responses reported in the literature are a late positivity called the P600 (Hagoort, Brown, \& Groothusen, 1993; Osterhout \& Holcomb, 1992), an early left anterior negativity (ELAN; Friederici, 2002; Neville, Nicol, Barss, Forster, \& Garrett, 
1991), and the syntactic mismatch negativity (sMMN; Pulvermüller \& Shtyrov, 2003; Shtyrov, Pulvermüller, Näätänen, \& Ilmoniemi, 2003). The latter response, sMMN, is particularly convenient because it has been elicited in orthogonal designs that rigorously control for effects of stimulus features of the critical words and their context, therefore making it possible to exclude important physical and psycholinguistic confounds. In addition, the mismatch negativity (MNN) is elicited independently of whether subjects attend to the input or not (Näätänen, 2001; Näätänen, Tervaniemi, Sussman, Paavilainen, \& Winkler, 2001) and therefore does not require that subjects perform a linguistic task. Note that a linguistic task such as, for example, well-formedness decision and even attentive listening may induce postlexical and postsyntactic processing differences between grammatical and ungrammatical strings (Pulvermüller \& Shtyrov, 2006). The syntactic MMN has therefore been proposed to reveal the largely attention-independent neuronal spreading of activity intimately related to instantaneous grammatical processing of an incoming string of words (Pulvermüller, Shtyrov, \& Ilmoniemi, 2003; Shtyrov et al., 2003).

To test predictions of the probabilistic and the discrete combinatorial approaches to the sequential ordering of words in sentences, we recorded the magnetic MNN (MNNm). The MMN has been known for some time as a brain reflex of acoustic change detection and, more recently, it has also been found to reflect the existence of cortical memory networks (Näätänen, 2001; Näätänen et al., 2001), including those engaged in grammar processing. Previous experiments have shown that the MMN to the same word is larger if this word is presented in an ungrammatical context, for example, if it does not show agreement with an adjacent word or produces a syntactic violation with a nonadjacent constituent in a context of several words (Menning et al., 2005; Pulvermüller \& Shtyrov, 2003; Shtyrov et al., 2003).

In the present experiment, the MNN was elicited by common well-formed word sequences and by strings that are uncommon and grammatically incorrect. As an additional condition, we used a string that was grammatically correct although highly uncommon, as revealed by statistical information about language use. ${ }^{1}$

For determining statistical properties of word strings, a measure similar to what Shannon and Weaver (1949) called mutual information was used. We calculated the frequency of occurrence or probability of a given string in standard text and divided this value by the product of the probabilities of each string segment. This led to a correlation measure, which we label normalized sequential probability (NSP; for further details, see Methods).

We elicited grammatical brain processes by word pairs that did agree with each other in grammatical gender, number, and case or did not agree in gender. Neurophysiological indicators of agreement have previously been described for various languages, including English (Pulvermüller \& Shtyrov, 2003; Osterhout, McKinnon, Bersick, \& Corey, 1996), Dutch (Hagoort \& Brown, 1999), German (Gunter, Friederici, \& Schriefers, 2000), Spanish (Barber \& Carreiras, 2003, 2005), Hebrew (Deutsch \& Bentin, 2001), and Finnish (Shtyrov et al., 2003). As the critical grammatical feature, we chose agreement between definite articles of German and their subsequent nouns. This makes it possible to record brain responses to the same target noun presented in determiner contexts where the noun is either common or rare and either grammatical or not, thus excluding confounds possibly related to physical and psycholinguistic differences between critical stimulus words (Pulvermüller \& Shtyrov, 2003; Shtyrov et al., 2003). More importantly, this experimental strategy even allows for a "crossed" design where the effects of determiner context and target noun are orthogonal to the effect of grammaticality or string probability (cf. Pulvermüller \& Shtyrov, 2006; Shtyrov et al., 2003). Common grammatical sequences were the singular nominative forms der Mut (the courage; singular, masculine gender, nominative case) and die Wut (the rage; singular, feminine gender, nominative and accusative case), which are characterized by high NSP ( $>1$ per million). The uncommon strings were die $M u t$, which is grammatically incorrect due to lack of gender agreement, and der Wut, which is grammatically correct (singular, feminine, genitive or dative case), but occurs only rarely in language use $(<1 / 4$ occurrence per million words; see Table 1). A general prediction is that grammatical violations elicit the syntactic MMN. Whereas the probabilistic approach predicts an enhanced MMN to uncommon grammatical strings, which approximates that of the uncommon ungrammatical ones, the discrete combinatorial theory predicts that the MMN elicited by critical well-formed but uncommon strings resembles that of highly frequent grammatical word combinations.

Table 1. Grammaticality and NSPs of Determiner-Noun Sequences Used in the Experiment

\begin{tabular}{|c|c|c|}
\hline \multirow[b]{2}{*}{ Standard Stimuli } & \multicolumn{2}{|c|}{ Deviant Stimuli } \\
\hline & $\begin{array}{l}\text { Wut }, f=49 \text { [fem, } \\
\text { nom] [fem, gen] }\end{array}$ & $\begin{array}{l}\text { Mut, } f=123 \\
\quad \text { [masc, nom] }\end{array}$ \\
\hline $\begin{array}{c}\operatorname{der}, f=82,938 \\
\text { [masc, nom }] \\
\text { [fem, gen }]\end{array}$ & $\begin{array}{l}\text { Agreement, low } \\
\text { NSP, } 0.25\end{array}$ & $\begin{array}{l}\text { Agreement, high } \\
\text { NSP, } 1.18\end{array}$ \\
\hline $\begin{array}{l}\text { die, } f=83,409 \\
{[\text { fem, nom }]}\end{array}$ & $\begin{array}{l}\text { Agreement, high } \\
\text { NSP, } 1.22\end{array}$ & $\begin{array}{l}\text { No agreement, } \\
\text { low NSP, } 0.00\end{array}$ \\
\hline \multicolumn{3}{|c|}{$\begin{array}{l}\text { Lexical frequencies and grammatical features of the individual stimulus } \\
\text { words used as standard and deviant stimuli in the experiment are sep- } \\
\text { arately listed at the left and top of the table (masc }=\text { masculine, fem }= \\
\text { feminine, nom = nominative, gen = genitive; } f=\text { lexical frequency). } \\
\text { NSP and lexical frequency values are given in occurrences per million } \\
\text { word pairs or words, respectively. }\end{array}$} \\
\hline
\end{tabular}




\section{METHODS}

\section{Subjects}

Thirteen subjects ( 7 women; mean age, 25 years), righthanded monolingual native speakers of German with at least 13 years of formal education, normal hearing, and no history of neurological disease or drug abuse, gave their informed consent and participated for a small honorarium. The study was approved by the Ethics Committee of the University of Konstanz.

\section{Stimuli}

Two German determiners der and die were chosen as standard stimuli, deviant stimuli were the nouns Wut (fury) and Mut (courage). The sequences die Wut, der Wut, and der Mut are grammatically correct noun phrases, whereas *die Mut is grammatically incorrect. The acoustic realizations of the words were synthesized using the MBROLA text-to-speech system (Dutoit, Pagel, Pierret, Bataille, \& van der Vreken, 1996). Subsequently, stimuli were adjusted so that they had the same fundamental frequency and maximal sound energy. The length of all standard stimuli was $250 \mathrm{msec}$ and that of all deviant stimuli $300 \mathrm{msec}$. The stimulus onset asynchrony was 500 msec.

Lexical frequencies for the stimulus words and word pair sequences were extracted from the Mannheim Corpus (Ortmann, 1975). Normalized sequential probabilities were calculated using a correlation measure, by using Equation 1:

$$
\mathrm{NSP}(\mathrm{AB})=f(\mathrm{AB}) /\{f(\mathrm{~A}) \times f(\mathrm{~B})\}
$$

where $\mathrm{A}$ and $\mathrm{B}$ are the first and second words in a string, $f(\mathrm{AB})$ is the frequency (number of occurrences in the corpus) of word sequence $\mathrm{AB}$, and $f(\mathrm{~A})$ and $f(\mathrm{~B})$ are the lexical frequencies of the individual words. Table 1 lists the NSP values for all stimulus materials.

\section{Procedure}

The experiment consisted of two blocks. In each block, both standard stimuli were each occurring with a probability of 0.42 . Each block included one of the deviant stimuli (either Wut or Mut) occurring with a probability of 0.16 . Block order was counterbalanced over subjects. The stimulus sequence was pseudorandomized with the following constraint: There were at least three standard stimuli between any two deviant stimuli and the first 30 stimuli were standards. Each deviant stimulus was presented 332 times, 166 times in each of the two local contexts (after der and die), which resulted in 2080 trials overall. Stimulation loudness was adjusted individually to a comfortable level (70-80 dB) and kept constant over both blocks. Subjects were instructed to focus their attention on a silent movie, which was played throughout the experiment, and to ignore the acoustic stimuli. No motor response was required and subjects were explicitly instructed to refrain from movements during the experiment as much as possible.

\section{Magnetoencephalography Recordings}

Neuromagnetic signals were recorded continuously with a 148-channel whole-head magnetometer (Magnes WHS 2500, 4D NeuroImaging Inc., San Diego, CA) using a 0.1to $100-\mathrm{Hz}$ pass band and sampled at a rate of $508 \mathrm{~Hz}$. Along with the magnetic signals the vertical and the horizontal electrooculogram (EOG) and the electrocardiogram were recorded. The head position was recorded by the Magnes coils to determine the position of the subjects' head relative to the sensors. These data together with the subjects' digitized head shape were used to fit the center of the spheres for source estimation (see below).

\section{Data Analysis}

Magnetoencephalography (MEG) data were submitted to a global noise filter subtracting the external, nonbiological noise obtained by the 11 MEG reference channels of the system. The data were then split into epochs, discarding all epochs with an EOG level $>100 \mu \mathrm{V}$ or MEG level $>5$ pT between minimum and maximum on one or more MEG channels. The first 30 trials of each block and the first standard trial after each deviant stimulus were also discarded. Two subjects had to be excluded due to excessive artifacts. For each remaining subject, each block, and each standard and each deviant stimulus in context, stimulus-triggered eventrelated magnetic fields were calculated relative to a 100-msec baseline.

Cortical sources of these averages were estimated using minimum norm estimation (MNE, Hauk, 2004; Hämäläinen \& Ilmoniemi, 1984). The MNE is an inverse method to reconstruct the topography of the primary current underlying a magnetic field distribution (Grave de Peralta Menendez, Hauk, Gonzalez Andino, Vogt, \& Michel, 1997) with an acceptable accuracy (here <5\%). During pseudoinversion, matrices were regularized (Tikhonov-Phillips, $\lambda=0.01$ ). Following Hauk and coworkers (Hauk, 2004; Hauk, Keil, Elbert, \& Muller, 2002), we approximated cortical activity in a three-dimensional source space consisting of four concentric spheres. The outer shell is individually fitted to the head shape of the subjects (4-D Neuroimaging software). Sarvas (1987) proved that the head radius is not relevant for the computation of the magnetic field generated by primary currents in a spherically symmetric volume conductor. We assumed the head radius to be $10 \mathrm{~cm}$ and will report the MNE for the shell at $80 \%$ radius, which roughly 
corresponds to the cortical surface in the brain. On this shell, 197 equidistant dipoles were assumed that were used for further analysis.

The MNN was computed by subtracting the brain response to the standard stimulus from that to the deviant stimulus (Näätänen, 1995). Subtraction of brain responses to standard stimuli from the brain responses to deviant stimuli was performed in source space (i.e., on the minimum norm estimate) at the single-subject level. Because the event-related fields and current sources elicited by the determiners der and die may differ, the source average over both was calculated for each block and subtracted from each of the deviant stimulus sources recorded in the same block. Calculating MMN sources in this way minimizes the possibility that any differences between multiple standard stimuli affect the results (Pulvermüller et al., 2001). Statistical analyses showed that the event-related fields and source constellations elicited by the same determiners presented in different blocks did not differ between each other (cf. Figure 1A, der $r_{W u t}$, in red vs. der $r_{M u t}$, in blue, and $d i e_{W u t}$, in green vs. die $_{M u t}$ in black). Responses to the determiners der and die differed between each other after 200 msec (see Results). The critical comparisons were made between MMN responses to the same noun stimulus presented in different contexts, so that acoustic, phonological, lexical, or semantic differences between critical noun stimuli could not become relevant.

MMN source estimates were submitted to statistical analysis. Overall source strength was compared between contexts and critical noun stimuli. In addition, regions of interest were defined in left and right posterior perisylvian cortex, centered around the superior temporal lobe where an earlier magnetic sMMN study had reported
Figure 1. Global mean of all sources underlying magnetic brain responses to spoken words in context. (A) Responses to standard stimuli, the determiners der and die followed by the two nouns, respectively. Differences between words started $>200$ msec after stimulus onset. There were no differences between contexts in which these words were presented. (B) Brain responses to deviant stimuli, the nouns Mut and Wut presented in different determiner contexts. Between 140 and 200 msec after its onset, the noun *Mut elicited an enhanced neuromagnetic response in the ungrammatical condition (after determiner die, black curve) compared with the grammatical context (after der, green curve). A similar context difference was absent for the noun Wut, which was grammatically correct in both contexts. (C) The magnetic equivalent of the MNN (MMNm), the signals to the noun deviant stimuli subtracted by those to article standard stimuli (see Methods). Note the clear syntactic enhancement of the magnetic MNN specifically to the ungrammatically placed noun (Mut after die, black curve).

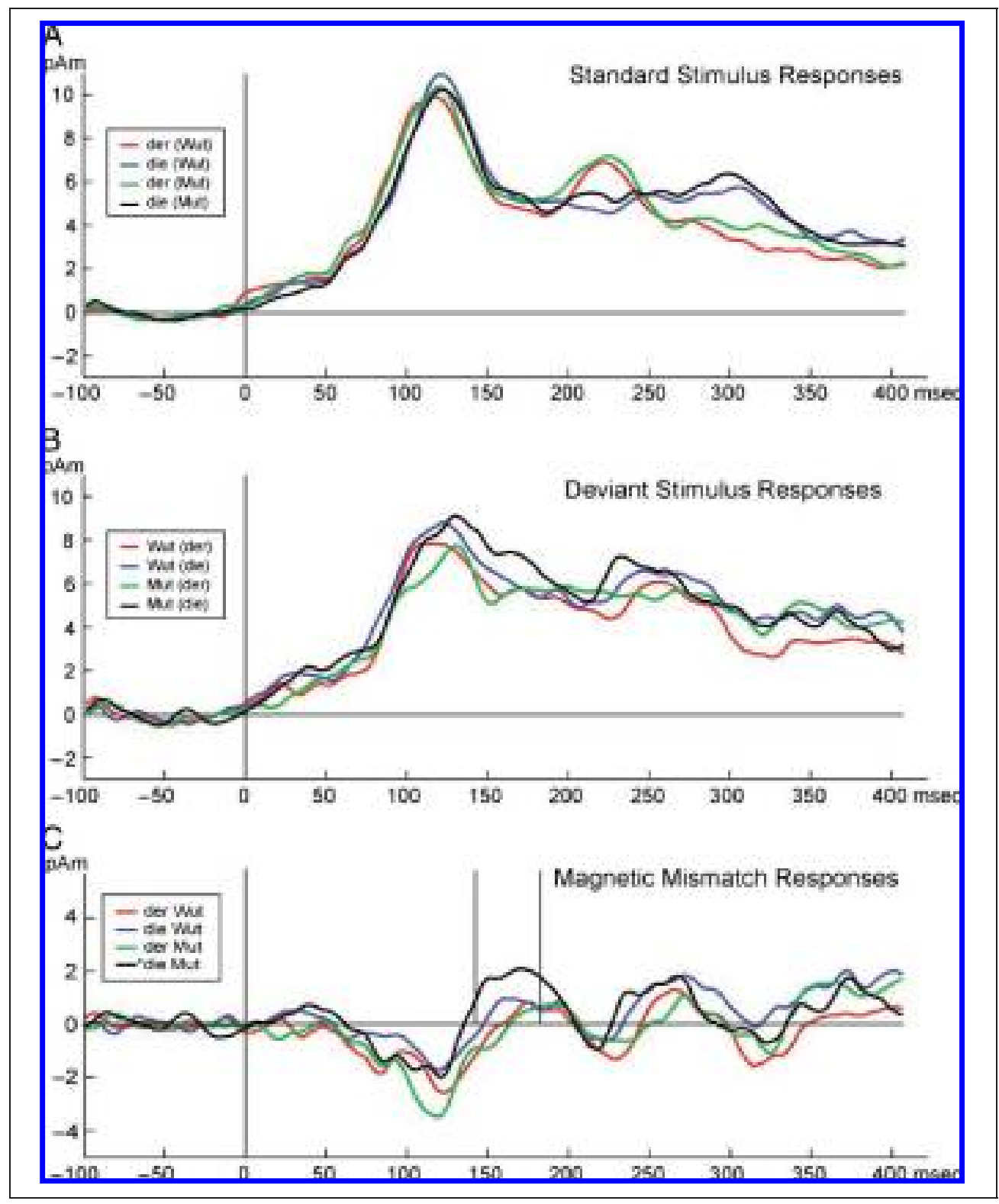


Table 2. Summary of Statistical Results of the Analysis of 40-msec Time Windows between 0 and $480 \mathrm{msec}$

\begin{tabular}{llrc}
\hline Time (msec) & \multicolumn{1}{c}{ Effect } & $F(1,10)$ & $p$ \\
\hline $120-160$ & Main effect: Hemisphere & 10.83 & $<.008^{*}$ \\
$160-200$ & Interaction: Context $\times$ & 5.30 & $<.04^{*}$ \\
& $\quad$ Noun & & \\
$200-240$ & Main effect: Context & 5.21 & $<.04^{*}$ \\
& Interaction: Context $\times$ & 6.64 & $<.02^{*}$ \\
& $\quad$ Hemisphere & & \\
$360-400$ & Interaction: Context $\times$ & 5.29 & $<.04^{*}$ \\
& Hemisphere & & \\
\hline
\end{tabular}

Post hoc tests on the Context $\times$ Noun interaction revealed that the incorrect phrase *die Mut differed from its correct counterpart der Mut, whereas there was no significant difference between the two correct phrases die Wut and der Wut.

significant grammaticality effects (Shtyrov et al., 2003). In addition, two frontocentral regions of interest were defined, as there is also evidence for inferior frontal sources of syntactically related brain activity (Friederici, Wang, Herrmann, Maess, \& Oertel, 2000). Because differential activation was predicted to be reflected in the MMN, statistical analyses were performed on average source strengths calculated for a time window capturing the peak of the MMN (140-180 msec). To obtain a broader picture of possible additional effects, further statistical analyses were performed for 40-msec-wide windows of the first $480 \mathrm{msec}$ following stimulus onset (significant results summarized in Table 2). Repeated measures analyses of variance (designs: Context $[$ der, die $] \times$ Noun $[$ Wut, Mut $]$ and Hemisphere [left, right $] \times$ Context $[$ der, die $] \times$ Noun $[W u t, M u t]$ ) were calculated to assess significance of between-category differences. $F$ tests were used to further investigate significant main effects or interactions.

\section{RESULTS}

Figure 1 shows global means of the estimated cumulative source strengths underlying the neuromagnetic responses to determiners (A) and nouns (B) of the noun phrases presented, along with those of the MMNms (C). Event-related fields to the two determiners diverged between each other after $>200$ msec following their onset, but there were no context-specific variations of source strengths. In contrast, noun responses appeared to diverge not only between stimulus words but also between contexts. Differences were already apparent before $200 \mathrm{msec}$ and were best visible in the MMNm difference waves (Figure 1C). Around 120 msec, source strengths were negative, indicating that responses to standard stimuli were stronger than those to deviant stimuli. MMNm sources then got positive and peaked around 160 msec. The peak amplitude was largest in the ungrammatical condition where determiner and noun did not agree in gender, case, and number (*die Mut, black trace), and equally small in all three grammatical conditions (plotted in red, green, and blue).

An additional peak in source strengths was visible around $270 \mathrm{msec}$, followed by another broad deflection starting around $340 \mathrm{msec}$. In contrast to the early MMNm peak at $160 \mathrm{msec}$, these two late waves did not show an obvious difference between conditions.

Figure 2 shows the distribution of sources underlying the MMNm, as obtained by the minimum norm estimate calculated for the 140- to $180-\mathrm{msec}$ time range. Compared to the syntactic violation condition, the syntactically correct phrases led to generally weaker MMNm sources. This difference seemed to be most pronounced in frontocentral areas in the left hemisphere. The frontal activity to grammatical phrases seemed to have its peak anterior to the main sources sparked by the ungrammatical phrase.

Statistical analyses of cumulative source strengths obtained for a window centered on the early peak of the MMNm (140-180 msec) revealed a significant interaction, $F(1,10)=5.34, p=.04$, between the factors Context (der, die) and Noun (Wut, Mut) (Figure 3). Planned comparison tests revealed a significant difference between grammatically correct and incorrect contexts for the noun Mut, $F(1,10)=5.14, p=.04$, but failed to document a significant difference between

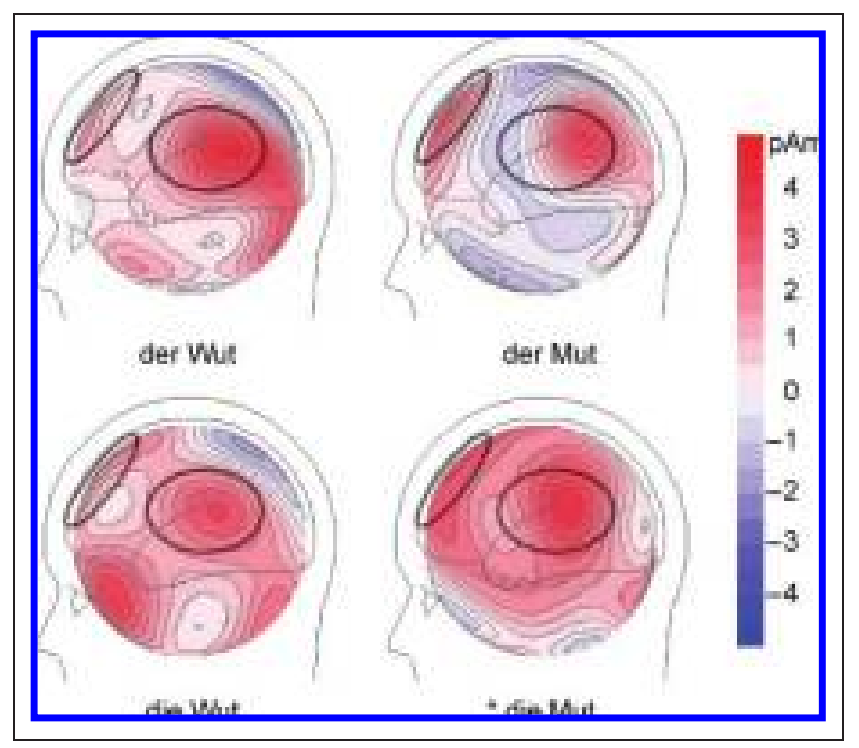

Figure 2. Estimated current sources underlying the magnetic MMN (MMNm) around $160 \mathrm{msec}$. Red colors indicate stronger activation to the deviant stimuli $(W u t, M u t)$ relative to standard stimuli, whereas blue colors denote the opposite, relatively stronger brain responses to the standard stimuli (der, die). A view on the left dominant hemisphere is shown for each noun in each context. Note the strong current sources in the ungrammatical condition, especially in frontal areas (*die Mut, lower right). Frontal and posterior perisylvian regions of interest are indicated by ellipses. 


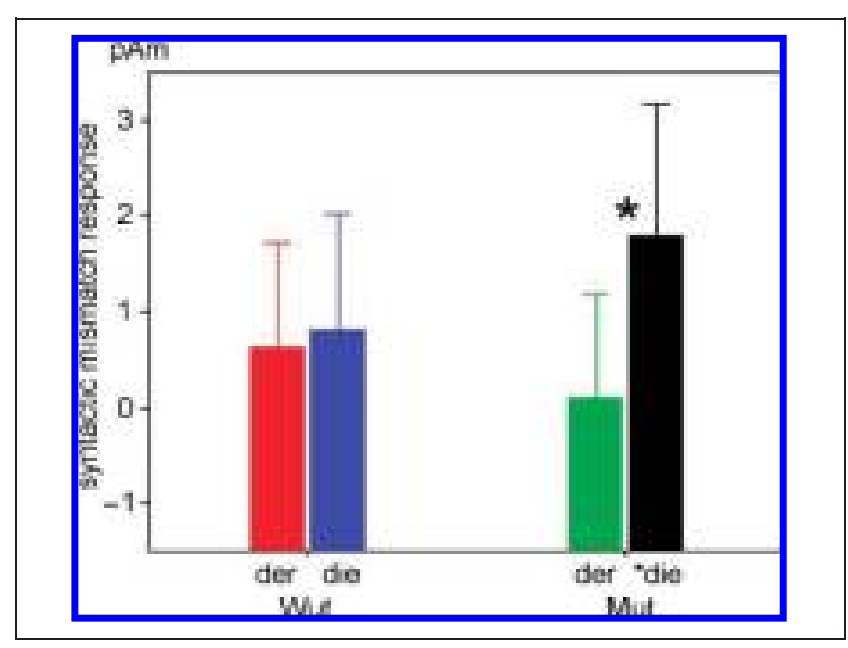

Figure 3. Means and standard errors of global MMNm source strengths. Around the peak of the MMN (140-180 msec), global MMNm sources were significantly stronger for ungrammatical strings compared with both common and uncommon grammatical strings.

presentations of the word Wut in grammatical common and grammatical uncommon context $(F=0.077, p>.7)$. Notably, there was no statistically significant difference between syntactically correct phrases or a main effect of determiner context. Analysis of responses to determiner standard responses (cf. Figure 1A) failed to show significant effects in the same time window, but "unsubtracted" responses to nouns (cf. Figure 1B) showed the same effect as the one revealed by the MMN difference, $F(1,10)=5.34, p=.04$. We do not interpret differences in the neurophysiological response to the determiners, as they could be related to acoustic differences between the stimulus words.

A systematic analysis was performed on consecutive time windows of 40-msec widths to further investigate possible differences of the mismatch response as a function of phrasal context and critical noun stimulus. Because earlier work indicated that dynamics of the syntactic modulation of the magnetic mismatch response are primarily carried by sources in the superior temporal cortex (Shtyrov et al., 2003), analyses focused on regions of interest in the left and right superior perisylvian cortex. The first significant differences were seen at 120-160 msec, where a main effect of laterality emerged, $F(1,10)=10.83, p=.008$, revealing stronger MMN sources in the left posterior perisylvian cortex than in the right.

The time frame 160-200 msec confirmed the results of the MMNm peak analysis, again revealing a significant interaction of the factors Noun and Context, $F(1,10)=$ $5.30, p=.04$, in the posterior perisylvian cortices. The difference between grammatical and ungrammatical context was again significant for the word Mut, $F(1,10)=$ $11.1, p=.007$, but a corresponding difference between common and uncommon grammatical phrases including the noun Wut was absent.
Further significant differences emerged at 200$240 \mathrm{msec}$, where a main effect of Context, $F(1,10)=$ $5.21, p=.04$ (stronger sources for die than for $d e r$ ), and an interaction of the factors Context and Hemisphere, $F(1,10)=6.64, p=.02($ der $>$ die, left $>$ right, laterality for die greater than for der), were significant. Finally, the window 360-400 msec gave evidence of another significant interaction of the factors Context and Hemisphere, $F(1,10)=5.29, p=.04$. In this case, the MMNm at posterior perisylvian sites after der tended to be stronger in the left hemisphere than in the right, whereas the reverse was true for the die context. These results are summarized in Table 2.

Further investigation of sources in the frontal and parietooccipital cortex failed to reveal significant effects, apart from the following: Frontal sources showed a main effect of Noun around the peak of the MMNm (160200 msec), $F(1,10)=5.84, p=.03$. Generally, frontal sources analyzed separately failed to reveal a main effect of Context or the critical interaction of the Context and Noun factors.

\section{DISCUSSION}

We asked whether neurophysiological brain responses reflect the sequential probability of spoken word strings or rather their grammatical correctness. Using a design controlling for differences between critical stimulus words, we looked at the sMMN, an early brain response previously suggested as an index of grammatical violations, which was, in the present experiment, elicited by noun phrases including a determiner and a noun. Determiner-noun sequences were either grammatically correct or not (agreement violation) and varied in the likelihood with which they occur in direct sequence in language use (NSP).

The sMMN, which peaked at $\sim 160$ msec after noun onset, revealed a significant interaction of the influence of determiner context and that of the critical stimulus, the noun. This interaction was due to a significant difference between the MMNs to grammatical and ungrammatical strings and to the absence of a similar difference between strings with low and high NSP. Average values even suggested a difference of mean values in the opposite direction (smaller average activation to uncommon grammatical strings than to common ones), thus arguing against the possibility that an uncommoncommon difference in the direction of the ungrammaticalgrammatical difference remained undetected due to lack of sensitivity. The main result, the significant interaction of context and critical word, was revealed by an overall analysis of all cortical sources underlying the MMNm and was confirmed by a region-of-interest analysis of posterior perisylvian sources.

These results confirm that the sMMN is driven by grammatical processes but do not provide direct sup- 
port for models implying that sequential probability of individual words is a crucial factor in sMMN elicitation. The present data are therefore best explained by an abstract combinatorial process that generalizes over both common and rare grammatical strings and distinguishes them from uncommon ungrammatical word sequences. In contrast, the present results are more difficult to explain in a framework postulating mechanisms that directly map sequential probabilities on neural circuits (see also discussion below, Kuhn \& Cruse, 2005; Elman et al., 1996; Willwacher, 1982).

\section{Early Neurophysiological Reflection of Agreement}

The present findings add to recent research documenting brain reflections of grammar (for a review, see Friederici, 2002). Following up on earlier work on the sMMN, we confirm here that this brain response, similar to the syntactic ELAN, reflects early syntactic processing. In contrast to the ELAN, the sMMN does not require application of linguistic and attention-demanding tasks and is even elicited in the absence of focused attention to speech. Both ELAN and SMMN peak within the first 100-200 msec after the relevant information is present in the input and indicate, among other grammatical phenomena, violations of rules of agreement. In the syntactic violation condition of the present experiment, the lack of agreement can be attributed to the (gender) feature, which would take the values (feminine) for die but (masculine) for Mut. The early neurophysiological manifestation of agreement violations is remarkable because most neurophysiological investigations of agreement reported longer latency brain correlates of this syntactic feature. Violations in number agreement were reported at $200-500 \mathrm{msec}$ after presentation of critical stimulus information (Osterhout \& Mobley, 1995; Kutas \& Hillyard, 1983) and even after 500 msec (Hagoort et al., 1993); in addition, gender agreement violations were reported at $200-500 \mathrm{msec}$ (Gunter et al., 2000; Osterhout \& Mobley, 1995) and later on (Barber \& Carreiras, 2005; Brown, van Berkum, \& Hagoort, 2000). Early brain indicators $(<250 \mathrm{msec})$ of syntactic agreement violations were first reported by Deutsch and Bentin (2001) (subject-predicate gender agreement in Hebrew) and the same result emerged from work using the sMMN (subject-predicate agreement in number and person in Finnish and English, Pulvermüller \& Shtyrov, 2003; Shtyrov et al., 2003). As, in the present data set, the sMMN enhancement was seen to violations of gender agreement between determiner and noun within a noun phrase, the evidence so far obtained suggests similar early brain mechanisms of agreement across languages and agreement types.

We should point out that, apart from the pioneering work by Friederici's and Hagoort's groups (e.g., Friederici, Pfeifer, \& Hahne, 1993; Hagoort et al., 1993) and a few who followed up on them, most earlier studies used written language materials to elicit syntactic brain responses. Speech stimuli pose a great challenge to neuroscience studies of syntax. As pointed out in earlier publications (e.g., Shtyrov et al., 2003), speech varies considerably in its physical characteristics and stimulus materials are therefore much more difficult to control than in studies using written language. In addition, physical and psycholinguistic variation between stimulus words may reduce the likelihood of recording reliable early brain responses. The reason for this is the following: In contrast to the late responses, which are widespread and long-lasting, the early responses are usually shortlived and topographically specific, so that any variance may annihilate them in the grand averages (Pulvermüller, 1999). The MMN design we applied here offers a unique possibility to reduce stimulus variance and exactly control for physical speech parameters because the brain responses to the same critical speech stimulus are investigated in different contexts (Pulvermüller \& Shtyrov, 2006).

\section{Topography of sMMN Generators}

We calculated minimum norm estimates of cortical sources underlying the MMNm to obtain clues about the brain areas possibly contributing to the syntactic effect. There was a centrofrontal focus reminiscent of the Friederici et al. (2000) frontal generator of the syntactic ELAN, which appeared to differentiate between grammatical and ungrammatical strings, although in the present data set grammatically related dynamics of frontal source could not be confirmed by statistical results (see Results). There is strong evidence that superior temporal activation relates to grammatical processing (Kaan \& Swaab, 2002), and, consistent with this, the present study showed activation differences between grammatical and ungrammatical strings in the posterior perisylvian cortex. In an earlier MEG study of the sMMN, Shtyrov et al. (2003) also reported a superior temporal main source of this brain response, whereas source localization performed on the sMMN recorded in electroencephalogram (EEG) experiments suggested an additional frontal source (Pulvermüller \& Shtyrov, 2003). It may therefore be that distributed frontotemporal generators underlie the sMMN and similar early syntactic brain responses. At present still not fully understood properties of experimental paradigms, recording methods and analysis procedures may determine their predominance at frontal or temporal perisylvian sites in different experiments. Left laterality of syntactic processes is suggested by the laterality effect observed at 120-160 msec and later on. In the posterior perisylvian cortex activity was stronger in the left hemisphere than in the right, a finding consistent with the wellknown laterality of syntax. Taken together, these results are consistent with the idea that neuronal assemblies 
distributed over perisylvian cortex and lateralized to the left hemisphere implement the cortical mechanisms of syntactic processing.

\section{Syntax Processing Outside the Focus of Attention}

It is most remarkable that MMN is still elicited under distraction, although the subjects' attention is directed away from the MMN-eliciting stimuli (Näätänen, 1995). In the present study, subjects had to watch a silent movie while stimuli were presented auditorily and tried to ignore the speech stimuli. The results may therefore appear to be in line with claims about the automaticity of syntax (Frazier, 1987; Fodor, 1983). However, we cannot fully exclude the possibility that, in the present paradigm, subjects did occasionally sneak a little listen to some of the speech stimuli (Carlyon, 2004), although we reassured ourselves by questioning subjects after the experiment that they did not actively listen. Thus, the brain response to the syntactic violation studied here was elicited in the absence of the subjects' constant focused attention to language stimuli. Therefore, it appears that basic matching processes at an early stage of syntax processing are computed outside the focus of attention.

It may be argued that, in spite of the task, which required subjects to ignore the stimuli and attend to a film, there could be a process by which subjects, after realizing that some of the strings are ungrammatical, direct their attention to ungrammatical strings, so that the MMN might become greater because of this redirection of attention. A number of reasons speak against this possibility: First, attention to violations predicts an enhanced P300 effect to these same items, too, and, as we have shown in previous work using event-related potentials and EEG, such P300 dynamics to grammatical violations do not occur in the MMN paradigm (Pulvermüller \& Shtyrov, 2003). There is also no indication in the present data that would suggest a neuromagnetic effect similar to the neuromagnetic equivalent of the P300 documented in earlier work (e.g., Kessler et al., 2005). Second, standard stimuli in the present experiment were pseudorandom sequences of determiners so that a general attention-to-violation mechanism would habituate. Third, no instruction was given to focus on grammatical violations and, as we did not employ any linguists or linguistics students in this experiment, it might be equally likely that they detect and then attend to uncommon (rather than ungrammatical) strings. Together, these points argue against the possibility that directed attention to violations might account for the results reported in this study.

\section{Theoretical Implications}

We interpret our main result as evidence for a discrete combinatorial system in the brain that operates on words and their affixes and responds differently to correct sentences and ungrammatical strings. Such a system has been postulated by linguistic theory (Steedman, 2000; Pinker, 1994; Chomsky, 1957). Although little is known about its neuronal implementation in the brain, the neural machinery realizing a combinatorial system should treat rare and common sentences in the same way, but should process strings that do not conform to the system's combinatorial principles differently. Even strings that have never been encountered before but conform to an abstract combinatorial rule should be processed in a similar fashion as a high-frequency string. This suggests combinatorial units connected with classes of neural word and morpheme representations. Such grammatical sequence detectors have been proposed to mediate syntactic priming between a word in the input and its possible successors in a string, so that a reduced brain response is elicited whenever a word sequence matches a syntactic rule. In contrast, an ungrammatical sequence will lead to the unprimed activation in the lexicosyntactic network, thus eliciting a larger early neurophysiological response (syntactic ELAN or MMN). This model may offer a basis for the explanation of neurophysiological correlates of syntactic processing (Knoblauch \& Pulvermüller, 2005; Pulvermüller, 2003a, 2003b; Pulvermüller \& Shtyrov, 2003). It accounts for the critical interaction observed in the present study and explains why rare and common sentences elicited a similar MMN, but only ungrammatical rare strings of spoken words were followed by a pronounced sMMN.

Although our results confirm predictions of a discrete combinatorial system account, they do not strictly falsify a probabilistic mapping perspective. A learning approach according to which NSP is mapped in a linear fashion on synaptic connection strength and the further assumption that this translates linearly into neurophysiological activation would make incorrect predictions on the outcome of this present experiment. However, such linear mappings are not necessary and actually not realistic. In a modified probabilistic framework, it is possible to postulate discreteness of the learning process, as the sequence detector model mentioned above posits (see Knoblauch \& Pulvermüller, 2005). A different type of argument relates to the nature of the MMN. It could be a specific feature of this brain response to reflect syntactic regularity but not NSP. Neuroscience research has produced evidence that the probability of co-occurrence of neuronal firing is mapped onto synaptic connection strength and it is therefore extremely unlikely that measures such as NSP are not reflected by the neural substrate. It seems advisable to accept probability mapping mechanisms but, at the same time, the accumulating evidence for discrete processing mechanisms of a rule-like type (Ullman, 2001). The present data would point to the potential importance of the rule construct even for explaining specific neurophysiological phenomena. 
Reprint requests should be sent to Friedemann Pulvermüller, MRC Cognition and Brain Sciences Unit, 15 Chaucer Road, Cambridge CB2 7EF, United Kingdom, or via e-mail: friedemann. pulvermuller@mrc-cbu.cam.ac.uk.

\section{Note}

1. The fourth theoretical possibility, frequently occurring but ungrammatical strings, does not exist, as a regularly used word string would per definitionem be in accord with the rules of a language.

\section{REFERENCES}

Barber, H., \& Carreiras, M. (2003). Integrating gender and number information in Spanish word pairs: An ERP study. Cortex, 39, 465-482.

Barber, H., \& Carreiras, M. (2005). Grammatical gender and number agreement in Spanish: An ERP comparison. Journal of Cognitive Neuroscience, 17, 137-153.

Brown, C. M., van Berkum, J. J., \& Hagoort, P. (2000). Discourse before gender: An event-related brain potential study on the interplay of semantic and syntactic information during spoken language understanding. Iournal of Psvcholinguistic Research, 29, 53-68.

Carlyon, R. P. (2004). How the brain separates sounds. Trends in Cognitive Sciences, 8, 465-471.

Chomsky, N. (1957). Syntactic structures. The Hague: Mouton.

Deutsch, A., \& Bentin, S. (2001). Syntactic and semantic factors in processing gender agreement in Hebrew: Evidence from ERPs and eye movements. Journal of Memorv and Language, 45, 200-224.

Dutoit, T., Pagel, V., Pierret, N., Bataille, F., \& van der Vreken, O. (1996). The MBROLA project: Towards a set of high-quality speech synthesizers free of use for non-commercial purposes. In Proceedings of the ICSLP (Vol. 3, pp. 1393-1396). Philadelphia.

Elman, J. L., Bates, L., Johnson, M., Karmiloff-Smith, A., Parisi, D., \& Plunkett, K. (1996). Rethinking innateness. A connectionist perspective on development. Cambridge: MIT Press.

Fodor, J. A. (1983). The modularity of mind. Cambridge: MIT Press.

Frazier, L. (1987). Sentence processing. In M. Coltheart (Ed.), Attention and performance XII: The psychology of reading (pp. 559-586). Hove, UK: Erlbaum.

Friederici, A. D. (2002). Towards a neural basis of auditory sentence processing. Trends in Cognitive Sciences, 6 , 78-84.

Friederici, A. D., Pfeifer, E., \& Hahne, A. (1993). Event-related brain potentials during natural speech processing: Effects of semantic, morphological and syntactic violations. Cognitive Brain Research, 1, 183-192.

Friederici, A. D., Wang, Y., Herrmann, C. S., Maess, B., \& Oertel, U. (2000). Localization of early syntactic processes in frontal and temporal cortical areas: A magnetoencephalographic study. Human Brain Mapping, 11, 1-11.

Grave de Peralta Menendez, R., Hauk, O., Gonzalez Andino, S., Vogt, H., \& Michel, C. (1997). Linear inverse solutions with optimal resolution kernels applied to the electromagnetic tomography. Human Brain Mabping, 5, 454-467.

Gunter, T. C., Friederici, A. D., \& Schriefers, H. (2000). Syntactic gender and semantic expectancy: ERPs reveal early autonomy and late interaction. Journal of Cognitive Neuroscience, 12, 556-568.
Hagoort, P., Brown, C., \& Groothusen, J. (1993). The syntactic positive shift (SPS) as an ERP-measure of syntactic processing. Language and Cognitive Processes, 8, 439-483.

Hagoort, P., \& Brown, C. M. (1999). Gender electrified: ERP evidence on the syntactic nature of gender processing. Journal of Psycholinguistic Research, 28, 715-728.

Hämäläinen, M. S., \& Ilmoniemi, R. J. (1984). Interpreting measured magnetic fields of the brain: Minimum norm estimates of current distributions (Tech. Rep. TKK-F-A559). Helsinki, Finland: Helsinki University of Technology.

Hauk, O. (2004). Keep it simple: A case for using classical minimum norm estimation in the analysis of EEG and MEG data. Neuroimage, 21, 1612-1621.

Hauk, O., Keil, A., Elbert, T., \& Muller, M. M. (2002). Comparison of data transformation procedures to enhance topographical accuracy in time-series analysis of the human EEG. Journal of Neuroscience Methods, 113, 111-122.

Kaan, E., \& Swaab, T. Y. (2002). The brain circuitry of syntactic comprehension. Trends in Cognitive Sciences, 6 , 350-356.

Kessler, K., Schmitz, F., Gross, J., Hommel, B., Shapiro, K., \& Schnitzler, A. (2005). Target consolidation under high temporal processing demands as revealed by MEG. Neuroimage, 26, 1030-1041.

Knoblauch, A., \& Pulvermüller, F. (2005). Sequence detector networks and associative learning of grammatical categories. In S. Wermter, G. Palm, \& M. Elshaw (Eds.), Biomimetic neural learning for intelligent robots (pp. 31-53). Berlin: Springer.

Kuhn, S., \& Cruse, H. (2005). Static mental representations in recurrent neural networks for the control of dynamic behavioural sequences. Connection Science, 17, 343-360.

Kutas, M., \& Hillyard, S. A. (1983). Event-related brain potentials to grammatical errors and semantic anomalies. Memory and Cognition, 11, 539-550.

Menning, H., Zwitserlood, P., Schoning, S., Hihn, H., Bolte, J., Dobel, C., et al. (2005). Pre-attentive detection of syntactic and semantic errors. NeuroReport. 16, 77-80.

Näätänen, R. (1995). The mismatch negativity: A powerful tool for cognitive neuroscience. Ear and Hearing, 16, 6-18.

Näätänen, R. (2001). The perception of speech sounds by the human brain as reflected by the mismatch negativity (MMN) and its magnetic equivalent (MMNm). Psychophysiology, 38, $1-21$.

Näätänen, R., Tervaniemi, M., Sussman, E., Paavilainen, P., \& Winkler, I. (2001). "Primitive intelligence" in the auditory cortex. Trends in Neurosciences, 24, 283-288.

Neville, H., Nicol, J. L., Barss, A., Forster, K. I., \& Garrett, M. F. (1991). Syntactically based sentence processing classes: Evidence from event-related brain potentials. Journal of Cognitive Neuroscience, 3, 151-165.

Ortmann, W. D. (1975). Hochfrequente deutsche Wortformen. Bde. 1 \& 2. München: Goethe-Institut, Arbeitsstelle für wissenschaftliche Didaktik.

Osterhout, L., \& Holcomb, P. J. (1992). Event-related brain potentials elicited by syntactic anomaly. Journal of Memory and Language, 31, 785-806.

Osterhout, L., McKinnon, R., Bersick, M., \& Corey, V. (1996). On the language specificity of the brain response to syntactic anomalies: Is the syntactic positive shift a member of the P300 family? Journal of Cognitive Neuroscience, 8, 507-526.

Osterhout, L., \& Mobley, L. A. (1995). Event-related brain potentials elicited by failure to agree. Iournal of Memory and Language, 34, 739-773.

Pinker, S. (1994). The language instinct. How the mind creates language. New York: Harper Collins.

Pulvermüller, F. (1999). Words in the brain's language. Behavioral and Brain Sciences, 22, 253-336. 
Pulvermüller, F. (2003a). The neuroscience of language. Cambridge: Cambridge University Press.

Pulvermüller, F. (2003b). Sequence detectors as a basis of grammar in the brain. Theorv in Biosciences, 122, 87-103.

Pulvermüller, F., Kujala, T., Shtyrov, Y., Simola, J., Tiitinen, H., Alku, P., et al. (2001). Memory traces for words as revealed by the mismatch negativity. Neuroimage, 14, 607-616.

Pulvermüller, F., \& Shtyrov, Y. (2003). Automatic processing of grammar in the human brain as revealed by the mismatch negativity. Neuroimage, 20, 159-172.

Pulvermüller, F., \& Shtyrov, Y. (2006). Language outside the focus of attention: The mismatch negativity as a tool for studying higher cognitive processes. Progress in Neurobiology, 79, 49-71.

Pulvermüller, F., Shtyrov, Y., \& Ilmoniemi, R. J. (2003). Spatio-temporal patterns of neural language processing: An MEG study using minimum-norm current estimates. Neuroimage, 20, 1020-1025.
Rogers, T. T., \& McClelland, J. L. (2004). Semantic cognition. A parallel distributed processing approach. Cambridge: MIT Press.

Sarvas, J. (1987). Basic mathematical and electromagnetic concepts of the biomagnetic inverse problem. Physics in Medicine and Biology, 32, 11-22.

Shannon, C. E., \& Weaver, W. (1949). The mathematical theory of communication. Urbana: University of Illinois Press.

Shtyrov, Y., Pulvermüller, F., Näätänen, R., \& Ilmoniemi, R. J. (2003). Grammar processing outside the focus of attention: An MEG study. Iournal of Cognitive Neuroscience, 15, 1195-1206.

Steedman, M. (2000). The syntactic process. Cambridge: MIT Press.

Ullman, M. T. (2001). A neurocognitive perspective on language: The declarative/procedural model. Nature Reviews Neuroscience, 2, 717-726.

Willwacher, G. (1982). Storage of a temporal pattern sequence in a network. Biological Cubernetics, 43, 115-126. 